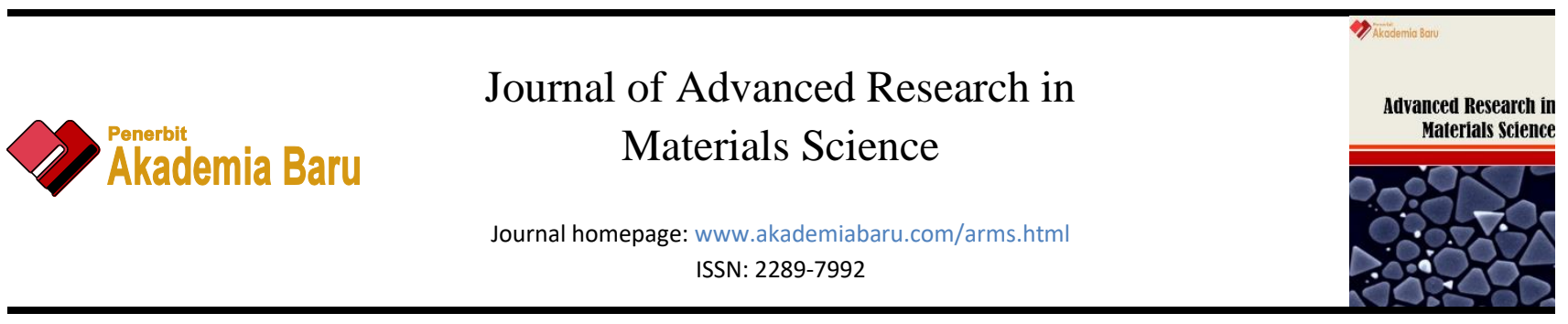

\title{
The Effects of Magnetic Casson Blood Flow in an Inclined Multi-stenosed Artery by using Caputo-Fabrizio Fractional Derivatives
}

\author{
Dzuliana Fatin Jamil ${ }^{1}$, Salah Uddin ${ }^{1}$, Rozaini Roslan ${ }^{1, *}$ \\ 1 Department of Mathematics and Statistics, Fakulti Sains Gunaan danTeknologi, Universiti Tun Hussein Onn Malaysia, 84600 Panchor, Johor, \\ Malaysia
}

\section{ARTICLE INFO}

ABSTRACT

\begin{abstract}
This paper investigates the magnetic blood flow in an inclined multi-stenosed artery under the influence of a uniformly distributed magnetic field and an oscillating pressure gradient. The blood is modelled using the non-Newtonian Casson fluid model. The governing fractional differential equations are expressed by using the fractional Caputo Fabrizio derivative without singular kernel. Exact analytical solutions are obtained by using the Laplace and finite Hankel transforms for both velocities. The velocities of blood flow and magnetic particles are graphically presented. It shows that the velocity increases with respect to the Reynolds number and the Casson parameter. Meanwhile, the velocity decreases as the Hartmann number increases. These results are useful for the diagnosis and treatment of certain medical problems.
\end{abstract}

Keywords:

Caputo Fabrizio derivative; Blood flow;

Magnetohydrodynamics; Multi-stenosis

Copyright $@ 2020$ PENERBIT AKADEMIA BARU - All rights reserved

\section{Introduction}

A detailed literature survey shows that cardiovascular disease is one of the fatal diseases currently. The main reason is due to the unusual hemodynamics within the bloodstream. Hemodynamic is the knowledge of blood circulation, which is useful in the diagnosis of coronary illness. The reason behind the malfunction of cardiovascular system is the presence of fats, cholesterol and lipoproteins at the sites of atherosclerotic lesion in the artery [1]. In recent years, due to its great importance in the human cardiovascular system, the study of blood flow through constricted arteries has received a great deal of attention [2-4]. Blood vessels have been regarded as having zero inclination, which were treated as horizontal in most available studies. Physically, these arteries are tilted [5]. Prasad and Radhakrishnamacharya [6] considered the steady blood flow through an inclined non-uniform tube with multiple stenoses. Agarwal and Varshney [7] studied the flow of Herschel-Bulkley fluid through an inclined tube of non-uniform cross-section with multiple stenoses. Biswas and Paul [8] observed the steady blood flow through an inclined tapered vessel, where the blood was modelled as Newtonian fluid and the slip vessel wall condition was applied.

\footnotetext{
* Corresponding author.

E-mail address: rozaini@uthm.edu.my

https://doi.org/10.37934/arms.72.1.1530
} 
Also, their analysis includes one-dimensional Poiseuille blood flow through tapered vessels with inclined geometries.

Extensive research has been done since the last few decades on the dynamics of biological fluid in the presence of magnetic field with implications in bio-engineering and medical technology. Bhargava et al., [9] investigated a mathematical model for blood flow through an inclined artery under the influence of an inclined magnetic field. Mekheimer et al., [10] discussed the blood flow through an elastic artery with overlapping stenosis under the effect of induced magnetic field. Many researchers considered blood as viscous and non-viscous fluid in stenotic arteries with magnetic field effects, but limited number of research works focused on the effect of induced magnetic field on blood flow through stenosis [11-12]. Mukhopadhyay and Layek [13] worked on a mathematical model to study blood flow through a variable shape stenosed artery under the influence of magnetic field and demonstrated the effect of stenosis shape and magnetic field on the flow resistance.

Plasma is classified as Newtonian fluid, but blood exhibits non-Newtonian behavior [14]. It is well known that blood being a suspension of cells behaves as a non-Newtonian fluid at low shear rate and while flowing through small blood vessels, especially in diseased states when clotting effects in small arteries are present [15]. As blood flows at low shear rate into narrow arteries, it behaves like a Casson fluid [16]. Many researchers [17-19] have been working on the Casson fluid model for modelling blood flow through narrow arteries. Nagarani and Sarojamma [20] studied the effect of body acceleration on pulsatile flow of Casson fluid through a mild stenosed artery. The pulsatile flow of Casson fluid with body acceleration subjected to a slip velocity condition in stenosed artery has been studied by Siddiqui et al., [21].

Caputo and Fabrizio gave a new expression for fractional derivative operator with an exponential kernel without singularities. Due to the increasing concern in modelling by using fractional derivatives, several fractional derivative models have been formulated by inferring the existing fluid models [22-23]. Ali et al., [24] had developed a fractional order model for blood flow (Casson fluid) with the help of Hankel and Laplace transform techniques to obtain the exact solutions. The so-called Caputo and Fabrizio fractional derivative is employed to solve different real problems [25]. Shah and Khan [26] applied the idea of the Caputo-Fabrizio fractional derivatives to generalize the starting flow of second grade fluid over a vertical plate and obtained the exact solutions using the Laplace transform technique. Saqib et al., [27] developed a mathematical model for MHD blood flow in a magnetite dusty particle tube by replacing the ordinary time derivative with a fractional time derivative of Caputo. Some other recent studies can be found in Alkahtani and Atangana [28], Shah et al., [29] and the references therein.

A thorough search of the relevant literature has witnessed the fact that the existing literature did not present the exact solution of MHD blood flow model in the context of Caputo Fabrizio fractional derivative for inclined multi-stenosed artery. In the present study, we use the fractional order time derivative to model the non-Newtonian Casson fluid. The blood flow is due to the oscillating pressure gradient in the $z$-direction and the external magnetic field. The fractional derivative model is obtained by converting the first-order derivatives to Caputo Fabrizio fractional derivatives for the axial blood flow and magnetic particles velocities. The exact solutions are then calculated by means of significant transformations like Laplace and finite Hankel transforms. For numerical computations, we take the zeros of the Bessel functions to generate the graphical findings by using Mathcad for different values of fractional parameters as well as some important physical parameters.

\section{Methodology}


Let us consider the unsteady blood flow in an inclined multi-stenosed artery as shown in Figure 1 aligned in the axial direction (z-axis). $r$-axis is the radial direction. Blood is treated as an incompressible non-Newtonian Casson fluid subjected to an oscillating pressure gradient. The blood flow is driven by an applied magnetic field and the induced magnetic field is assumed as negligible as it is considerably small as compared to the applied magnetic field. The corresponding momentum equation is therefore a generalization of the preview study conducted by Shah et al., [30] with the adding factors of Casson fluid in the inclined multi-stenosed artery. At $t=0$, the blood and the magnetic particles are treated as stationary.

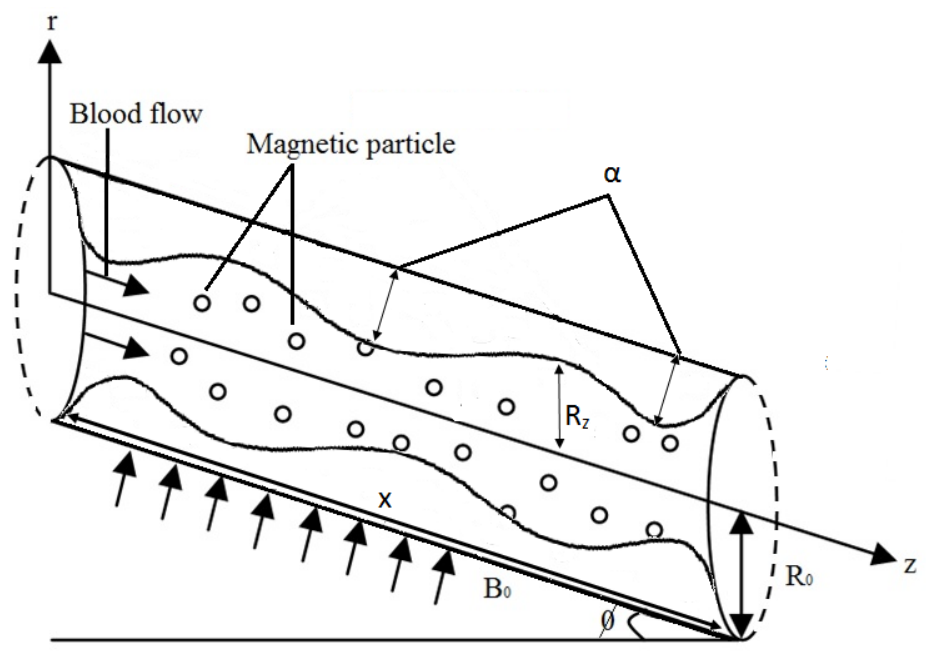

Fig. 1. Geometry of an inclined arterial segment with multi-stenosis.

The governing equations are the Navier-Stokes equations describing the blood flow, the Maxwell's relations describing the magnetic field and the Newton's second law describing the particle motion.

The Maxwell equations are

$\nabla \cdot \vec{B}=0, \nabla \times \vec{B}=\mu_{0} \vec{J}, \nabla \times \vec{E}=-\frac{\partial \vec{B}}{\partial t}$

where $\vec{B}$ is the magnetic flux intensity, $\mu_{0}$ is the magnetic permeability, $\vec{E}$ is the electric field intensity and $\vec{J}$ is the current density given by Zaman et al., [5] and Hatami et al., [31].

$\vec{J}=\sigma(\vec{E}+\vec{V} \times \vec{B})$

Here $\sigma$ is the electrical conductivity and $\vec{V}$ is the velocity field. The electromagnetic force $\vec{F}_{\text {em }}$ is defined as

$\vec{F}_{e m}=\vec{J} \times \vec{B}=\sigma(\vec{E}+\vec{V} \times \vec{B}) \times \vec{B}=-\sigma B_{0}^{2} u(r, t) \vec{k}$

where $\vec{k}$ is the unit vector of the $z$-direction and $\vec{V}=u(r, t) \vec{k}$ is the axial velocity of the blood. The force $\vec{F}_{e m}$ will be included in the momentum equations. 
The unsteady blood flow in an axisymmetric cylindrical tube of radius $R_{0}$ under the influence of uniform transverse magnetic field and pressure gradient of the form [7].

$-\frac{\partial p}{\partial z}=A_{0}+A_{1} \cos (\omega t), \quad A_{0}>0$.

is considered, where the constants $A_{0}$ and $A_{1}$ are the amplitudes of the pulsatile magnetic field and pressure gradient that give rise to systolic or diastolic pressure.

The geometry of the multi-stenosis in the arterial lumen may be described mathematically [11] as follows

$R_{z}=1-\alpha\left(1.48 z-0.7398 z^{2}+0.1485 z^{3}-0.013955 z^{4}+0.0006145 z^{5}-0.000010243 z^{6}\right)$

where $R_{z}$ is the radius of the artery in the constricted region, $R_{0}$ is the radius of the normal artery, $x$ is the length of stenosis and $\alpha$ is the degree of the stenosis.

The momentum equation for fluid flow in an inclined stenosed artery $[24,30,32]$ can be written as

$\frac{\partial u}{\partial t}=-\frac{1}{\rho} \frac{\partial p}{\partial z}+v\left(1+\frac{1}{\beta}\right)\left(\frac{\partial^{2} u}{\partial r^{2}}+\frac{1}{r} \frac{\partial u}{\partial r}\right)+\frac{K N}{\rho}(v-u)-\frac{\sigma B_{0}^{2} \sin \theta}{\rho} u+g \sin \phi$,

where $\rho$ is the fluid density, $v$ is the kinematic viscosity, $\beta=\frac{\mu_{B} \sqrt{2 \pi_{c}}}{\tau_{r}}$ is the material parameter of Casson fluid, in which $\mu_{B}$ is the plastic dynamic viscosity, $\tau_{r}$ is the yield stress of fluid, and $2 \pi_{c}$ is the critical value of this product based on the non-Newtonian model, $p$ is the pressure, $N$ is the number of magnetic particles per unit volume, $K$ is the Stokes constant, $u$ is the fluid velocity and $v$ is the velocity of the particle. The term $\frac{K N}{\rho}(v-u)$ is the force due to the relative motion between fluid and magnetic particles. It is assumed that the Reynolds number of the relative velocity is small. As such, the force between the magnetic particles and the blood is proportional to the relative velocity.

The motion of magnetic particles is governed by the Newton's second law:

$m \frac{\partial v}{\partial t}=K(u-v)$

where $m$ is the average mass of the magnetic particles.

In order to consider the time-fractional model, we firstly multiply Eqs. (Error! Reference source not found.) and Error! Reference source not found.) multiplied by $\lambda=\sqrt{\frac{R_{0} \rho}{A_{0}}}$, to yield a term with dimension of time t. The governing equations of the time-fractional model are 


$$
\begin{aligned}
\lambda^{\alpha} D_{t}^{\alpha} u & =-\frac{\lambda}{\rho}\left(A_{0}+A_{1} \cos (\omega t)\right)+\lambda v\left(1+\frac{1}{\beta}\right)\left(\frac{\partial^{2} u}{\partial r^{2}}+\frac{1}{r} \frac{\partial u}{\partial r}\right)+\frac{K N \lambda}{\rho}(v-u) \\
& -\frac{\sigma B_{0}^{2} \sin \theta \lambda}{\rho} u+g \lambda \sin \phi
\end{aligned}
$$

and

$$
\lambda^{\alpha} D_{t}^{\alpha} v=\frac{K \lambda}{m}(u-v)
$$

where the Caputo-Fabrizio derivative operator is

$$
{ }^{C F} D_{t}^{\alpha} u(r, t)=\frac{1}{1-\alpha} \int_{0}^{t} \exp \left(-\frac{\alpha(t-\tau)}{1-\alpha}\right) \frac{\partial u(r, \tau)}{\partial \tau} d \tau
$$

The Laplace transform of the Caputo-Fabrizio time derivative can be written as

$$
L\left\{{ }^{C F} D_{t}^{\alpha} u(r, t)\right\}=\frac{s L\{u(r, t)\}-u(r, 0)}{(1-\alpha) s+\alpha}
$$

The initial boundary conditions of the fluid inside the cylindrical domain of radius $R_{0}$ are

$$
\begin{array}{ll}
u(r, 0)=0, & v(r, 0)=0, \quad \text { at } r \in\left[0, R_{z}\right] \\
u(r, t)=0, & v(r, t)=0, \quad \text { at } r=R_{z}
\end{array}
$$

For dimensionless study, the following non-dimensional parameters can be introduced

$$
\begin{gathered}
r^{*}=\frac{r}{R_{0}}, t^{*}=\frac{t}{\lambda}, u^{*}=\frac{u}{u_{0}}, v^{*}=\frac{v}{u_{0}}, \\
A_{0}^{*}=\frac{\lambda A_{0}}{\rho u_{0}}, A_{1}^{*}=\frac{\lambda A_{1}}{\rho u_{0}}, \omega^{*}=\lambda \omega, g^{*}=\frac{g}{u_{0}^{2} / R_{0}}
\end{gathered}
$$

where $u_{0}$ is the characteristics velocity.

By introducing the above parameters and dropping the * notation the non-dimensional forms of Eqs. Error! Reference source not found.), Error! Reference source not found.), and Error! Reference source not found.) are

$$
D_{t}^{\alpha} u=A_{0}+A_{1} \cos (\omega t)+\beta_{1}\left[\frac{\partial^{2} u}{\partial r^{2}}+\frac{1}{r^{*}} \frac{\partial u^{*}}{\partial r^{*}}\right]+R(v-u)-H a^{2} u+\frac{\sin \phi}{F}
$$

$G \cdot D_{t}^{\alpha} v=u-v$ 
where $\operatorname{Re}=\frac{R_{z}^{2}}{\lambda v}$ is the Reynolds number, $R=\frac{k N \lambda}{\rho}$ is the particles concentration parameter,

$H a=B_{0} \sqrt{\lambda} \sqrt{\frac{\sigma}{\rho} \sin \theta}$ is the Hartmann number, $F=\frac{R_{0}}{\lambda u_{0} g}$ is the inclination angle parameter and $\beta_{1}=\frac{1}{\operatorname{Re}}\left[1+\frac{1}{\beta}\right]$.

The non-dimensional boundary conditions are

$$
\begin{aligned}
& u\left(\frac{r}{R_{z}}, 0\right)=0, \quad v\left(\frac{r}{R_{z}}, 0\right)=0, \quad \text { at } \frac{r}{R_{z}} \in[0,1] \\
& u\left(\frac{r}{R_{z}}, t\right)=0, \quad v\left(\frac{r}{R_{z}}, t\right)=0, \text { at } \frac{r}{R_{z}}=1
\end{aligned}
$$

Laplace transform is well suited when the temporal variable $t$ is adopted in the blood flow model (see Eqs. Error! Reference source not found.), Error! Reference source not found.)) and boundary condition in Eq. Error! Reference source not found.). After the transformation process, we obtain

$$
\begin{aligned}
& \frac{s \bar{u}(r, s)}{s+\alpha(1-s)}=\frac{A_{0}}{s}+\frac{A_{1} s}{s^{2}+\omega^{2}}+\beta_{1}\left[\frac{\partial^{2} \bar{u}(r, s)}{\partial r^{2}}+\frac{1}{r} \frac{\partial \bar{u}(r, s)}{\partial r}\right]+R \bar{v} \\
& -\left(R+H a^{2}\right) \bar{u}(r, s)+\frac{\sin \phi}{s F} \\
& G \frac{s \bar{v}(r, s)}{s+\alpha(1-s)}=\bar{u}(r, s)-\bar{v}(r, s) \\
& \bar{u}(1, s)=0, \quad \bar{v}(1, s)=0 .
\end{aligned}
$$

From Eq. Error! Reference source not found.), the following equation can be obtained:

$$
\bar{v}(r, s)=\frac{s+\alpha(1-s)}{G s+s+\alpha(1-s)} \bar{u}(r, s)
$$

Substituting $\bar{v}(r, s)$ from Eq. Error! Reference source not found.) into Eq. (Error! Reference source not found.), the following can be obtained:

$$
\begin{array}{r}
{\left[\frac{s}{s+\alpha(1-s)}-R\left(\frac{s+\alpha(1-s)}{s+s G+\alpha(1-s)}\right)+R+H a^{2}\right] \bar{u}(r, s)=} \\
\frac{A_{0}}{s}+\frac{A_{1} s}{s^{2}+\omega^{2}}+\beta_{1}\left[\frac{\partial^{2} \bar{u}}{\partial r^{2}}+\frac{1}{r} \frac{\partial \bar{u}}{\partial r}\right]+\frac{\sin \phi}{s F}
\end{array}
$$


Applying finite Hankel transform of order zero (i.e. applying the boundary condition in Eq. Error! Reference source not found.) in Eq. (Error! Reference source not found.)) the following equation can be obtained:

$$
\begin{array}{r}
{\left[\frac{s}{s+\alpha(1-s)}-R\left(\frac{s+\alpha(1-s)}{s+s G+\alpha(1-s)}\right)+R+H a^{2}\right] \bar{u}_{H}\left(r_{n}, s\right)=} \\
{\left[\frac{A_{0}}{s}+\frac{A_{1} s}{s^{2}+\omega^{2}}+\frac{\sin \phi}{s F}\right] \frac{J_{1}\left(r_{n}\right)}{r_{n}}-\beta_{1} r_{n} \bar{u}_{H}\left(r_{n}, s\right)}
\end{array}
$$

where $\bar{u}_{H}\left(r_{n}, s\right)=\int_{0}^{1} r \bar{u}(r, s) J_{0}\left(r_{n} r\right) d r$ represents the finite Hankel transform of the velocity function $\bar{u}(r, s)=L T[u(r, t)]$ and $r_{n}, n=1,2, \ldots$ are the positive roots of the equation $J_{0}(x)=0$, Here $J_{0}$ is the Bessel function of order zero and it belongs to the first kind. By simplifying the coefficient of $\bar{u}_{H}\left(r_{n}, s\right)$ in Eq. (Error! Reference source not found., the following equations can be formulated:

$$
\begin{aligned}
\bar{u}_{H}\left(r_{n}, s\right) & =\frac{s^{2} y_{5 n}+s y_{6 n}+\alpha^{2}}{s^{2} y_{2 n}+s y_{3 n}+y_{4 n}}\left[\frac{1}{s}\left(A_{0}+\frac{\sin \phi}{F}\right)+\frac{A_{1} s}{s^{2}+\omega^{2}}\right] \frac{J_{1}\left(r_{n}\right)}{r_{n}} \\
\bar{u}_{H}\left(r_{n}, s\right)= & {\left[\frac{y_{9 n}}{s-y_{7 n}}+\frac{y_{10 n}}{s-y_{8 n}}\right]\left[\frac{1}{s}\left(A_{0}+\frac{\sin \phi}{F}\right)+\frac{A_{1} s}{s^{2}+\omega^{2}}\right] \frac{J_{1}\left(r_{n}\right)}{r_{n}} } \\
\bar{u}_{H}\left(r_{n}, s\right)= & \left(A_{0}+\frac{\sin \phi}{F}\right)\left[\frac{s^{-1}}{s-x_{7 n}} y_{9 n}+\frac{s^{-1}}{s-x_{8 n}} y_{10 n}\right] \frac{J_{1}\left(r_{n}\right)}{r_{n}} \\
& +A_{1} \frac{s}{s^{2}+\omega^{2}}\left[y_{9 n} \frac{1}{s-x_{7 n}}+y_{10 n} \frac{1}{s-x_{8 n}}\right] \frac{J_{1}\left(r_{n}\right)}{r_{n}}
\end{aligned}
$$

Note, the parameters in Eqs. (Error! Reference source not found.) and (Error! Reference source not found.) introduced for simplifying the coefficient of $\bar{u}_{H}\left(r_{n}, s\right)$ are:

$$
\begin{gathered}
\mathrm{y}_{1 n}=H a^{2}+R+\beta_{1} r_{n}^{2}, \\
y_{2 n}=1+G-\alpha-R-R \alpha^{2}+2 R \alpha+y_{1 n}+\alpha^{2} y_{1 n}-2 \alpha y_{1 n}+G y_{1 n}-G \alpha y_{1 n}, \\
y_{3 n}=\alpha+2 R \alpha^{2}-2 R \alpha-2 x_{1 n} \alpha^{2}+2 \alpha x_{1 n}+G \alpha x_{1 n}, \quad \mathrm{y}_{4 n}=\alpha^{2} y_{1 n}-R \alpha^{2}, \\
\mathrm{y}_{5 n}=1+\alpha^{2}-2 \alpha+G-G \alpha, \quad \mathrm{y}_{6 n}=-2 \alpha^{2}+2 \alpha+G \alpha,
\end{gathered}
$$




$$
\begin{array}{ll}
\mathrm{y}_{7 n}=\frac{-y_{3 n}+\sqrt{y_{3 n}^{2}-4 y_{2 n} y_{4 n}}}{2 y_{2 n}}, & \mathrm{y}_{8 n}=\frac{-y_{3 n}-\sqrt{y_{3 n}^{2}-4 y_{2 n} y_{4 n}}}{2 y_{2 n}}, \\
\mathrm{y}_{9 n}=\frac{y_{7 n}^{2} y_{5 n}+y_{7 n} y_{6 n}+\alpha^{2}}{y_{7 n}-y_{8 n}}, & \mathrm{y}_{10 n}=\frac{y_{8 n}^{2} y_{5 n}+y_{8 n} y_{6 n}+\alpha^{2}}{y_{8 n}-y_{7 n}},
\end{array}
$$

The Laplace transform of the image function $\bar{u}_{H}\left(r_{n}, s\right)$ discussed in Eq. (Error! Reference source not found.) can be obtained by using the Robotnov and Hartley's functions:

$$
\begin{gathered}
L T^{-1}\left[\frac{1}{s^{w}+y}\right]=F_{w}(-y, t)=\sum_{n=0}^{\infty} \frac{(-y)^{n} t^{(n+1) w-1}}{\Gamma((n+1) w)}, w>0 \\
L T^{-1}\left[\frac{s^{z}}{s^{w}+y}\right]=R_{w, z}(-y, t)=\sum_{n=0}^{\infty} \frac{(-y)^{n} t^{(n+1) w-1-z}}{\Gamma((n+1) w-z)}, \operatorname{Re}(w-z)>0 \\
\bar{u}_{H}\left(r_{n}, t\right)=\frac{J_{1}\left(r_{n}\right)}{r_{n}}\left[\left(e^{y_{7 n} t}-1\right)\left(\frac{A_{0} y_{9 n}}{y_{7 n}}+\frac{y_{9 n} \sin \phi}{y_{7 n} F}\right)+\left(e^{y_{8 n} t}-1\right)\left(\frac{A_{0} y_{10 n}}{y_{8 n}}+\frac{y_{10 n} \sin \phi}{y_{8 n} F}\right)\right. \\
\left.+A_{1} y_{9 n} e^{y_{7 n} t} * \cos (\omega t)+A_{1} y_{10 n} e^{y_{8 n} t} * \cos (\omega t)\right]
\end{gathered}
$$

By inverting the finite Hankel transforms (i.e. Eq. (Error! Reference source not found.)) we obtain

$$
\begin{gathered}
u(r, t)=2 \sum_{n=1}^{\infty} \frac{J_{0}\left(\frac{r}{R_{z}} r_{n}\right)}{r_{n} J_{1}^{2}\left(r_{n}\right)} \times u_{H}\left(r_{n}, t\right) \\
\begin{aligned}
u(r, t)=2 \sum_{n=1}^{\infty} \frac{J_{0}\left(\frac{r}{R_{z}} r_{n}\right)}{r_{n} J_{1}\left(r_{n}\right)}\left[\left(e^{x_{7 n} t}-1\right)\left(\frac{A_{0} y_{9 n}}{y_{7 n}}+\frac{y_{9 n} \sin \phi}{y_{7 n} F}\right)+\left(e^{y_{8 n} t}-1\right)\left(\frac{A_{0} y_{10 n}}{y_{8 n}}+\frac{y_{10 n} \sin \phi}{y_{8 n} F}\right)\right. \\
\left.\quad+A_{1} y_{9 n} e^{y_{7 n} t} * \cos (\omega t)+A_{1} y_{10 n} e^{y_{8 n} t} * \cos (\omega t)\right]
\end{aligned}
\end{gathered}
$$

The magnetic particle velocity can then be obtained from Eq. Error! Reference source not found.):

$$
\begin{aligned}
& \bar{v}(r, s)=\frac{s+\alpha-\alpha s}{s+G s+\alpha-\alpha s} \bar{u}(r, s) \\
& v(r, t)=x_{12 n}\left(1-y_{11 n}\right)\left[u(r, t) * e^{y_{12 n} t}\right], \quad 0<\alpha<1
\end{aligned}
$$

In Eqs. Error! Reference source not found. and Error! Reference source not found.), $f * g$ represents the convolution product of $f$ and $g$. The parameters introduced in Eq. Error! Reference source not found.) are: 
$y_{11 n}=\frac{1-\alpha}{G-\alpha+1}, \quad \mathrm{y}_{12 n}=\frac{\alpha}{G-\alpha+1}$

The convolution product of $f$ and $g$ can be calculated as

$$
(f * g)(t)=\int_{0}^{t} f(\tau) g(t-\tau) d \tau
$$

\section{Results and Discussions}

In order to determine the impact of the different flow parameters on the blood flow velocity $u(r$, $t$ ) and the magnetic velocity $v(r, t)$, we have implemented a Mathcad code to obtain the numerical results extracted from the analytical solutions in Eqs. (31) and (33). The fractional parameters result on the velocity distributions are presented graphically. The velocity results for several nondimensional parameters such as Reynolds number $R e$, Casson fluid parameter $\beta$, and Hartmann number $\mathrm{Ha}$ are presented in Figures 2-8.

For numerical computations, the following values are set as [24,30] $A_{0}=0.5, A_{1}=0.6, G=$ $0.8, R=0.5, R e=3, \omega=\frac{\pi}{4}, H a=2$ and $\beta=0.4$. All velocity profiles have been plotted for different fractional parameters and $r$ values. It is interesting to note that the fractional parameter plays a key role in regulating blood velocity. Here we use $\alpha=0.4,0.6,0.8,1$. Fractional derivatives describe memory effects, so fractional models provide additional information on the behavior of fluids with complex rheology as compared to ordinary models. The numerical results have been compared with [30] for having the same blood flow with magnetic particles as shown in Figure 2 . In the current work, we concentrate on the Casson fluid with magnetic particles that flow through an inclined multiple stenosed artery while [30] considered the blood flow with magnetic particles travelling through a cylindrical tube under the influence of a magnetic field and an oscillating pressure gradient. We have set $A_{0}=0.5, A_{1}=0.1, G=0.8, R=0.5, \quad R e=5, \omega=\frac{\pi}{4}, H a=2, z=$ 1 and $\beta=0.25$ for comparison purposes, so that both problems become similar.

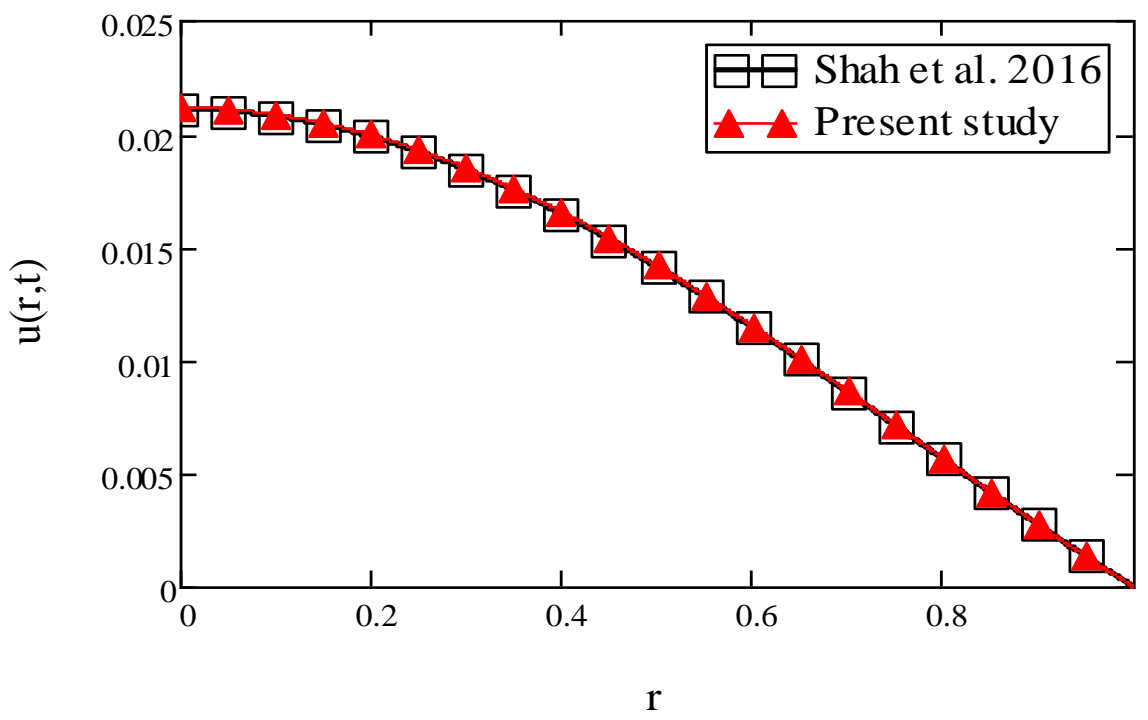

Fig. 2. Comparison of velocity distribution with previous study

Blood flow resistance is a factor controlling the blood flow rate. For both profiles, Figure 3 illustrates the velocity distributions at different locations: $z=0.5,1.5,3,5$ for representing various 
stenotic regions. From this figure it is found that the blood flow movement is slower as blood passes through the narrow stenotic region as compared to that in the wider part. It can be found that the patterns of flow resistance in both regions are similar for different stenotic sizes. It is important to note that increased stenotic height will increase flow resistance as the blood vessel diameter becomes smaller when the blood flows at vessel section of radius 0.2 . It is indeed significant to mention that the fractional parameter changes the velocity profiles entirely.
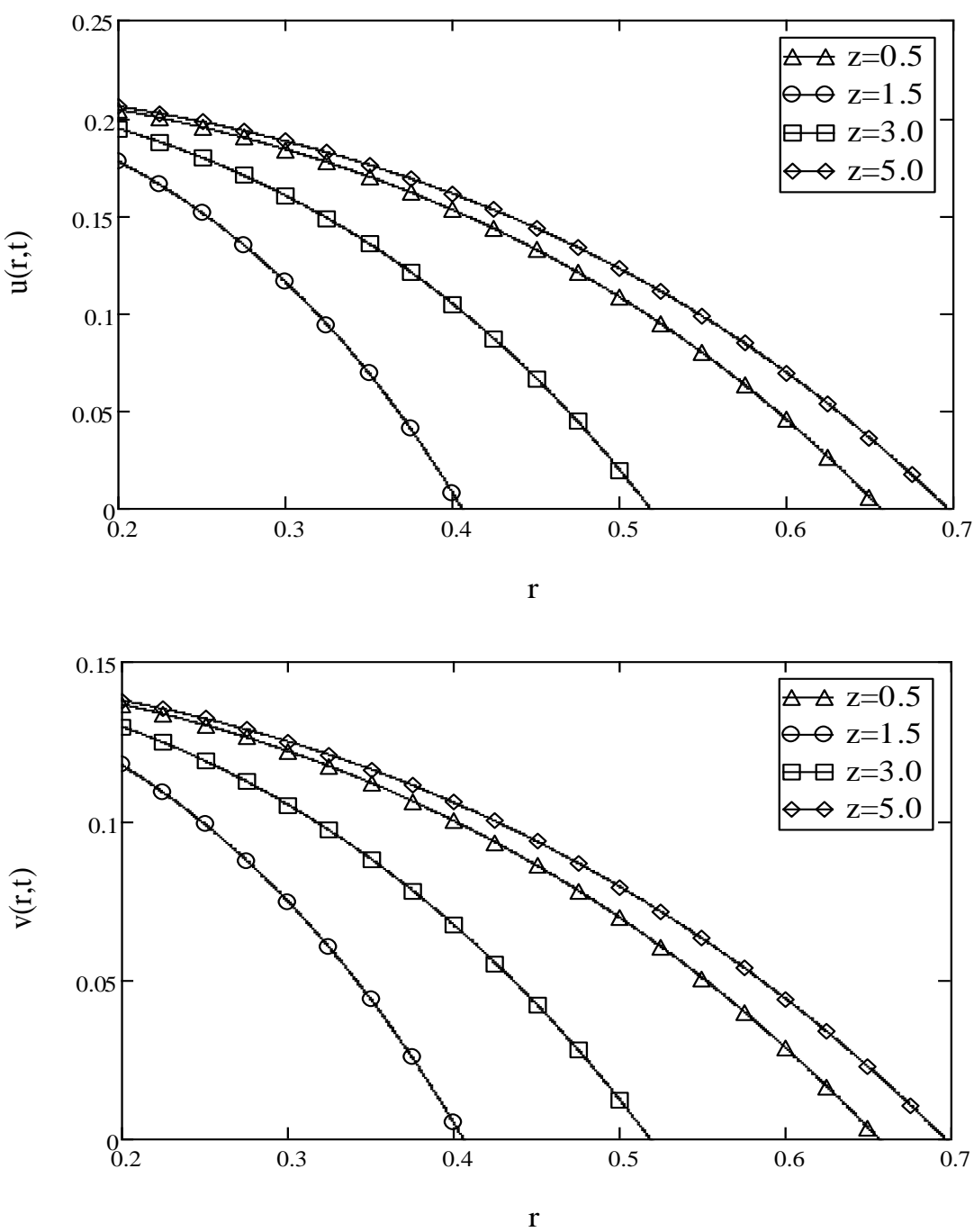

Fig. 3. Axial velocity profiles $u(r, t)$ and $v(r, t)$ at different values of $z$

Figure 4 is plotted to analyze the impacts of the fractional parameter on the blood flow and magnetic particle velocities. Also, the velocity components for various fractional parameter values $\alpha=0.4,0.6,0.8$ and for the ordinary fluid corresponding to $\alpha=1$ are sketched in these figures. It indicates that the blood modelled using the ordinary model flows faster than that using the Casson fluid model with fractional derivatives. This study illustrates the effectiveness of fractional-derivative models. By choosing an appropriate value of the fractional parameter $\alpha$, a more physical flow field 
can be obtained which in some cases may be considered more suitable for a particular practical problem.

Figure 5 represents the velocity profiles of blood and magnetic particles at three different time levels, i.e. $t=0.25,0.35,0.45$ with three different values of fractional parameter $\alpha=0.6,0.8,1$. In this figure the influence of fractional parameter has been shown for different time levels and it is observed that the velocity for the integer order fluid is higher than that for the fractional order derivative fluid for both profiles. The blood velocity increases with respect to time. The increment pattern is similar for the first two fractional parameter models, but the gap at different time levels is getting smaller for the ordinary model. However, in the case of the distribution of magnetic particles, an opposite phenomenon is observed, i.e. velocity increases as time decreases. The fractional parameter is found to play a key role in regulating the blood distributions. Figure 6 shows the velocity profiles for different Reynolds numbers $R e$. It is clear that the Reynolds number is proportionally related to the velocity of the fluid. In general, increase in Reynolds number will increase the velocity of the fluid gradually. Physically, lower viscosity (increased velocity) will increase $R e$. This figure shows the influence of the fractional parameter and it is observed that the velocity at the central axis increases with respect to fractional parameter.
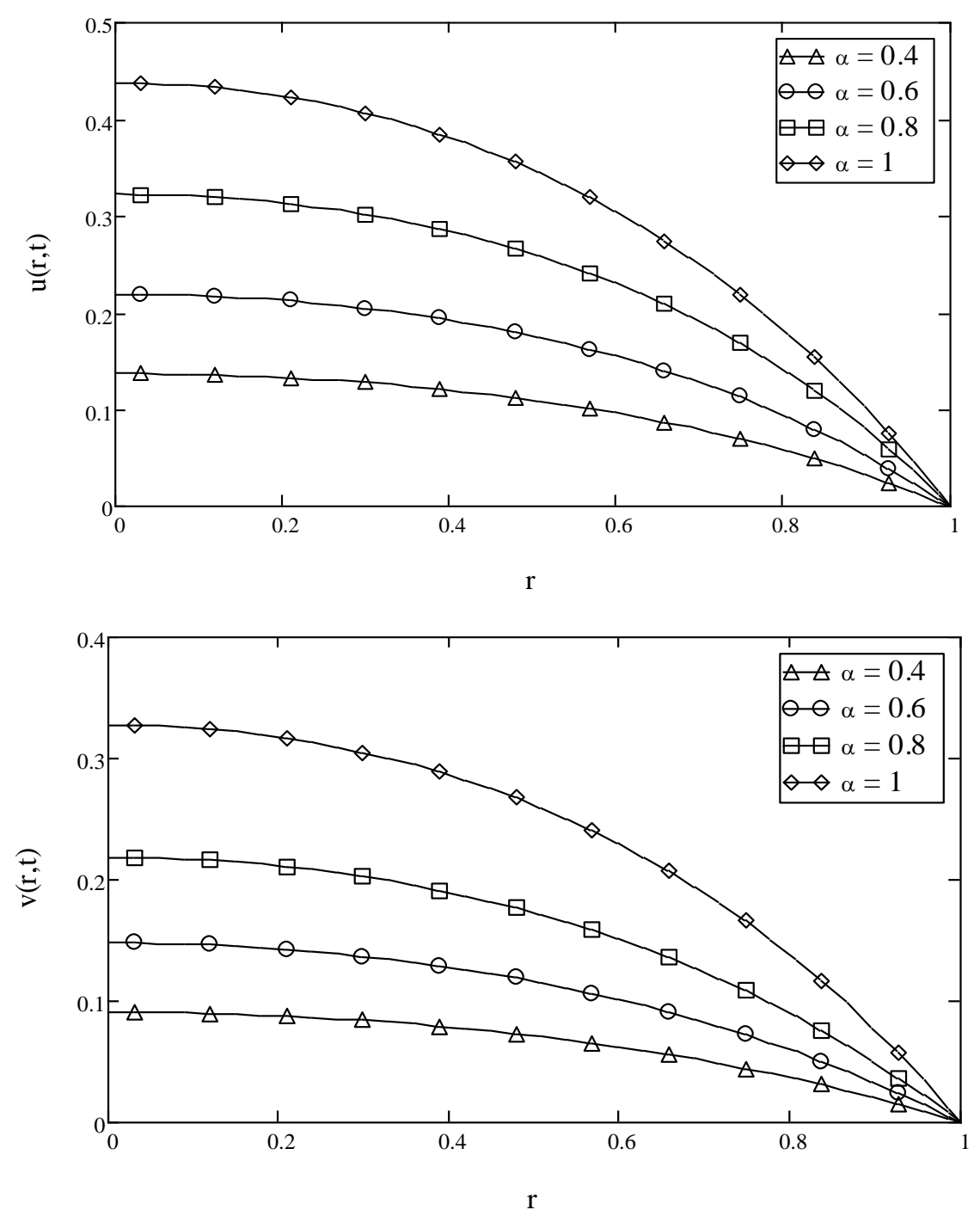

Fig. 4. Axial velocity profiles $u(r, t)$ and $v(r, t)$ for different fractional parameters 
The Casson parameter is related to the non-Newtonian nature of the blood. Higher Casson parameter is attributed to the Newtonian nature. The effects of Casson fluid parameter on the blood and magnetic particle motions are depicted in Figure 7. With an increase in the Casson fluid parameter, the fluid velocity increases. Casson nature is more significant for small arteries where red blood cells (RBCS) can accumulate due to rotation near the axis of the artery, creating a region depicted in the cells. This statement is in perfect agreement with Ali et al., [33] for a horizontal cylinder. It is hypothesized that the yield stress declines as $\beta$ increases and the thickness of the boundary layer decreases. The magnetic field is used for regulating the blood flow within the human circulatory system.
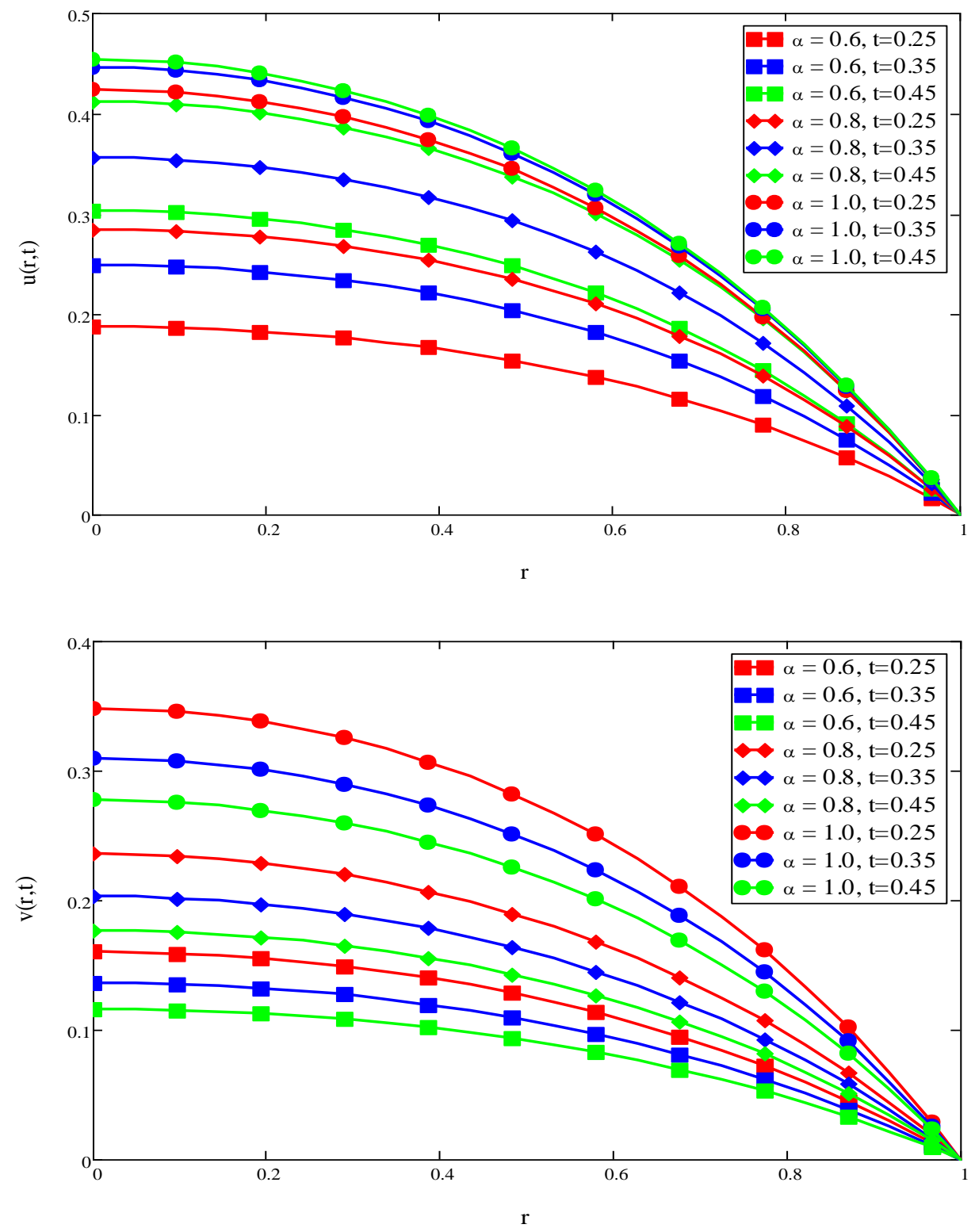

Fig. 5. Axial velocity profiles $u(r, t)$ and $v(r, t)$ for different time levels $t$

The effects of magnetic parameter on both fluid and magnetic particle velocities are shown in Figure 8. By increasing the Hartmann number $\mathrm{Ha}$, the blood velocity decreases. It is noticeable that the magnetic field will reduce the axial velocities of blood and magnetic particles substantially. Meanwhile, under the influence of magnetic field, the charged particles would undergo rotational 
motion. The action of magnetic orientation would induce further the suspension of red blood cells and magnetic particles. Higher concentration of magnetic particles will also increase the internal viscosity of the blood, thereby raising the rate of axial flow. The usefulness of the magnetic field in the fluid flow model would increase the Lorentz force, thus restricting the blood flow in the system.
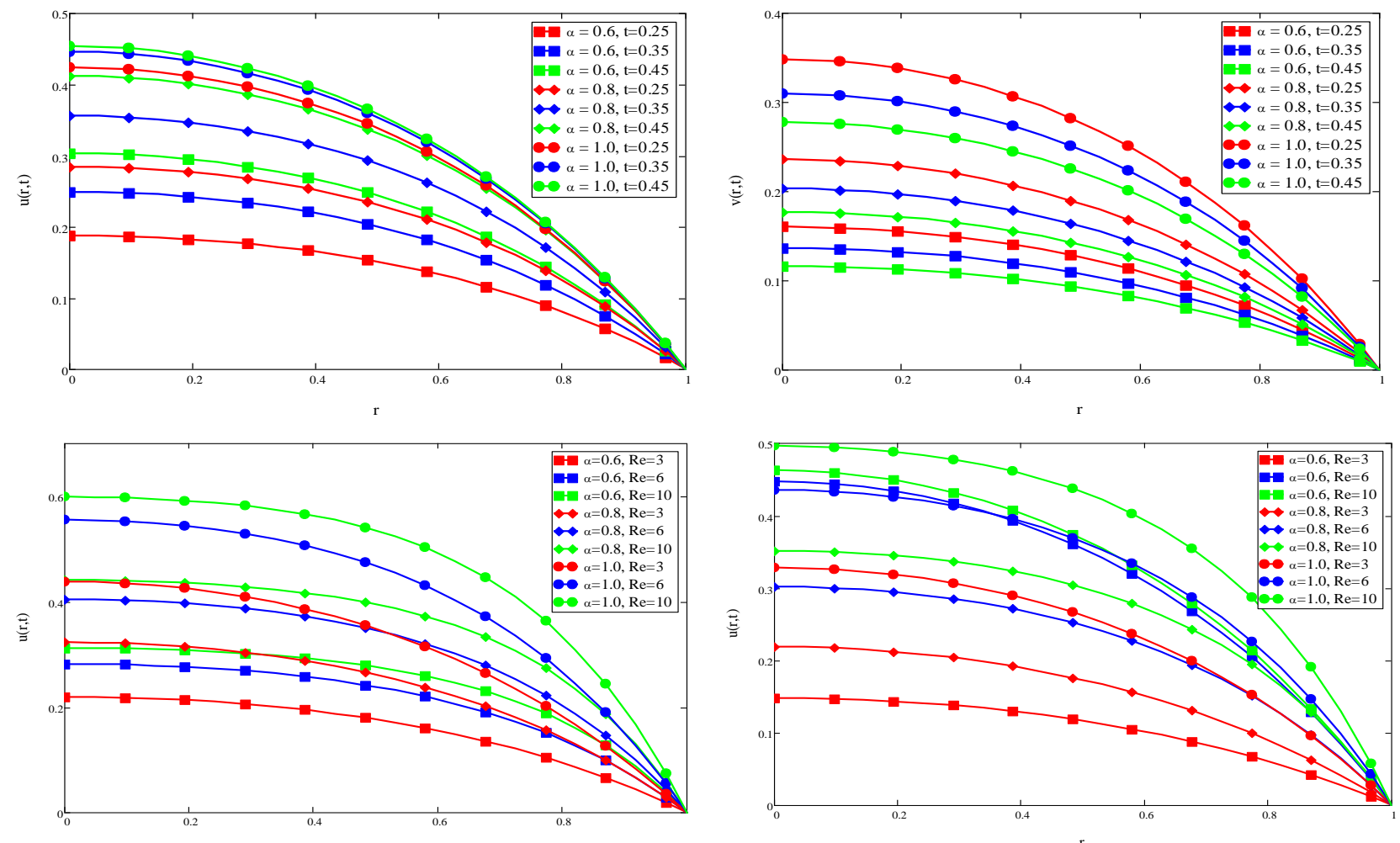

Fig. 6. Axial velocity profiles $u(r, t)$ and $v(r, t)$ for different Reynolds numbers
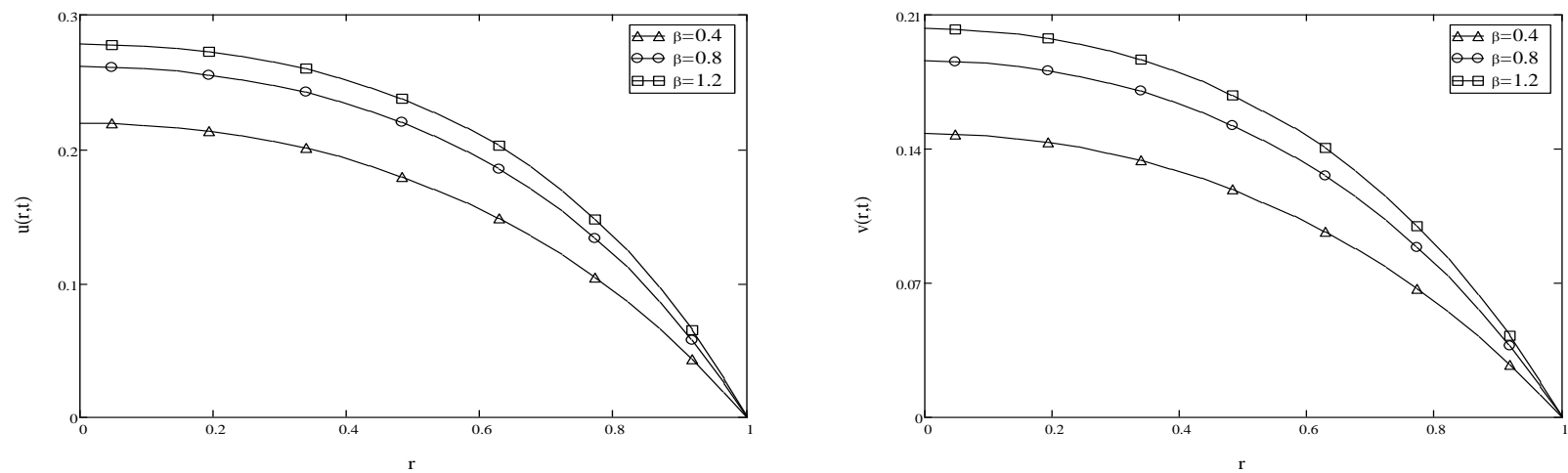

Fig. 7. Axial velocity profiles $u(r, t)$ and $v(r, t)$ for different Casson fluids 

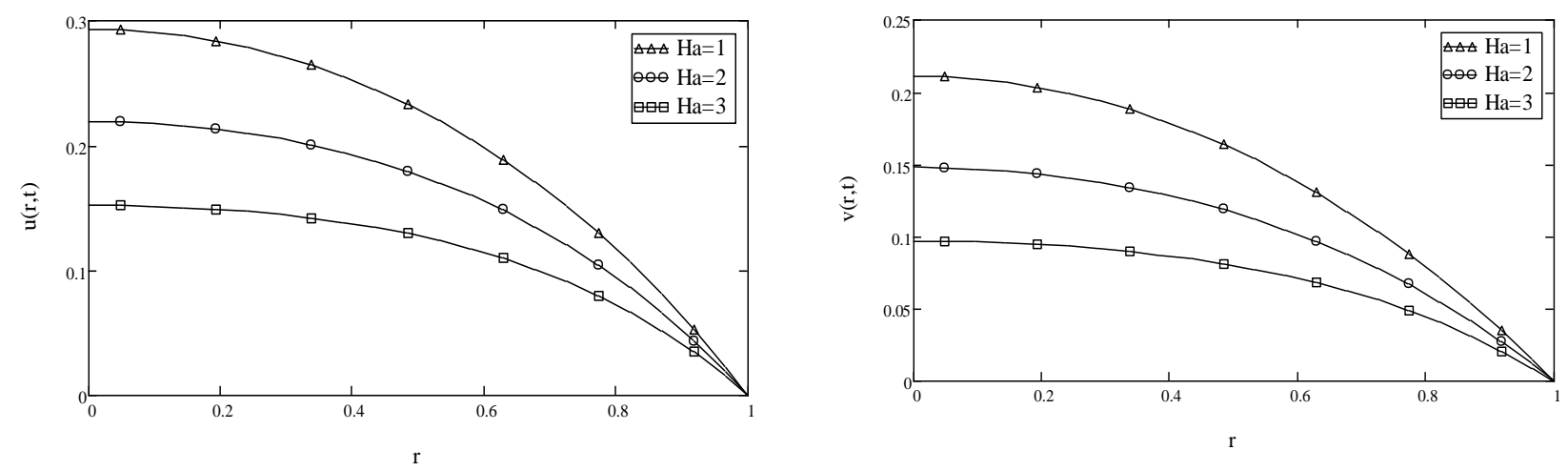

Fig. 8. Axial velocity profiles $u(r, t)$ and $v(r, t)$ for different Hartmann numbers

\section{Conclusions}

A mathematical analysis on the fractional order blood flow model under the influence of an external magnetic field acting on the non-Newtonian Casson fluid that flows through a multistenosed artery has been performed. The Caputo Fabrizio time fractional derivative has been used to obtain the exact solutions by the use of the Laplace and finite Hankel order zero transformation. Typically, an additional mathematical solution is needed to extract the ordinary model; however, the ordinary model $(\alpha=1)$ for the velocity equation can be obtained directly by using the current method since the equation is completely compatible. We have obtained the solutions of the problem based on the above transformations performed on the boundary conditions. The blood flow and magnetic particles distributions are highly influenced by the fractional order parameter. It should be noted that the particle has the same tendency as the blood; however, it moves slower. The blood velocity increases with respect to Reynolds number. In the meantime, the increase in Casson fluid parameters increases the velocities of blood and particles. On the other hand, the blood velocity decreases with respect to Hartmann number. These findings will be beneficial for atherosclerosis therapy.

\section{Acknowledgement}

This research was funded by grants from Research Management Centre University Tun Hussein Onn Malaysia under grant TIER 1/H072.

\section{References}

[1] Majee, Sreeparna, and G. C. Shit. "Numerical investigation of MHD flow of blood and heat transfer in a stenosed arterial segment." Journal of Magnetism and Magnetic Materials 424 (2017): 137-147. https://doi.org/10.1016/j.jmmm.2016.10.028

[2] Singh, Sapna, and Rajeev Ratan Shah. "A numerical model for the effect of stenosis shape on blood flow through an artery using power-law fluid." Advances in Applied Science Research 1, no. 1 (2010): 66-73.

[3] Mathur, Pankaj, and Surekha Jain. "Pulsatile flow of blood through a stenosed tube: effect of periodic body acceleration and a magnetic field." Journal of biorheology 25, no. 1-2 (2011): 71-77. https://doi.org/10.1007/s12573-011-0040-5

[4] Ponalagusamy, R., and S. Priyadharshini. "Pulsatile MHD flow of a Casson fluid through a porous bifurcated arterial stenosis under periodic body acceleration." Applied Mathematics and Computation 333 (2018): 325-343. https://doi.org/10.1016/j.amc.2018.03.103

[5] Zaman, Akbar, Nasir Ali, and M. Sajid. "Slip effects on unsteady non-Newtonian blood flow through an inclined catheterized overlapping stenotic artery." Aip Advances 6, no. 1 (2016): 015118. https://doi.org/10.1063/1.4941358

[6] Maruthi Prasad, K., and G. Radhakrishnamacharya. "Flow of Herschel-Bulkley fluid through an inclined tube of nonuniform cross-section with multiple stenoses." Archives of Mechanics 60, no. 2 (2008): 161-172.

[7] Agarwal, Raja, and N. K. Varshney. "Pulsatile Flow of Herschel-Bulkley Fluid through an Inclined Multiple Stenoses Artery with Periodic Body Acceleration." Pelagia Research Library, Advances in Applied Science Research 7, no. 3 (2016): 102-113. 
[8] Biswas, Devajyoti, and Moumita Paul. "Study of blood flow inside an inclined non-uniform stenosed artery." International Journal of Mathematical Archive 4, no. 5 (2013): 33-42.

[9] Bég, O. Anwar, Tasveer A. Bég, R. Bhargava, S. Rawat, and D. Tripathi. "Finite element study of transient pulsatile magneto-hemodynamic non-Newtonian flow and drug diffusion in a porous medium channel." Journal of Mechanics in Medicine and Biology 12, no. 04 (2012): 1250081.

https://doi.org/10.1142/S0219519412500819

[10] Mekheimer, Kh S., M. H. Haroun, and M. A. Elkot. "Effects of magnetic field, porosity, and wall properties for anisotropically elastic multi-stenosis arteries on blood flow characteristics." Applied Mathematics and Mechanics 32, no. 8 (2011): 1047.

https://doi.org/10.1007/s10483-011-1480-7

[11] Tashtoush, Bourhan, and Ahmad Magableh. "Magnetic field effect on heat transfer and fluid flow characteristics of blood flow in multi-stenosis arteries." Heat and Mass transfer 44, no. 3 (2008): 297-304. https://doi.org/10.1007/s00231-007-0251-x

[12] Kumar, S., M. K. Sharma, K. Singh, and N. R. Garg. "MHD two-phase blood flow through an artery with axially nonsymmetric stenosis." International Journal of Mathematical Sciences \& Engineering Applications (IJMSEA) 5 (2011): 63-74.

[13] Mukhopadhyay, S., and G. Layek. "Numerical modeling of a stenosed artery using mathematical model of variable shape." Applications and Applied Mathematics: An International Journal 3, no. 2 (2008): 308-328.

[14] Johnston, Barbara M., Peter R. Johnston, Stuart Corney, and David Kilpatrick. "Non-Newtonian blood flow in human right coronary arteries: steady state simulations." Journal of biomechanics 37, no. 5 (2004): 709-720. https://doi.org/10.1016/i.jbiomech.2003.09.016

[15] Nagarani, P., and G. Sarojamma. "Flow of a Casson fluid through a stenosed artery subject to periodic body acceleration." In Proceedings of the 9th WSEAS intern. Conf. Mathematical and computational methods in science and engineering, pp. 237-244. 2007.

[16] Nagarani, P., G. Sarojamma, and G. Jayaraman. "Exact analysis of unsteady convective diffusion in Casson fluid flow in an annulus-Application to catheterized artery." Acta mechanica 187, no. 1-4 (2006): 189-202. https://doi.org/10.1007/s00707-006-0316-9

[17] Maiti, S., S. Shaw, and G. C. Shit. "Caputo-Fabrizio fractional order model on MHD blood flow with heat and mass transfer through a porous vessel in the presence of thermal radiation." Physica A: Statistical Mechanics and its Applications 540 (2020): 123149.

https://doi.org/10.1016/i.physa.2019.123149

[18] Misra, Jagadis Chandra, Sudi D. Adhikary, and Gopal Chandra Shit. "Mathematical analysis of blood flow through an arterial segment with time-dependent stenosis." Mathematical Modelling and Analysis 13, no. 3 (2008): 401 412. https://doi.org/10.3846/1392-6292.2008.13.401-412

[19] Sankar, D. S., and Usik Lee. "Nonlinear mathematical analysis for blood flow in a constricted artery under periodic body acceleration." Communications in Nonlinear Science and Numerical Simulation 16, no. 11 (2011): 4390-4402. https://doi.org/10.1016/i.cnsns.2011.03.020

[20] Nagarani, P., and G. Sarojamma. "Effect of body acceleration on pulsatile flow of Casson fluid through a mild stenosed artery." Korea-Australia Rheology Journal 20, no. 4 (2008): 189-196.

[21] Siddiqui, S. U., N. K. Verma, Shailesh Mishra, and R. S. Gupta. "Mathematical modelling of pulsatile flow of Casson's fluid in arterial stenosis." Applied Mathematics and Computation 210, no. 1 (2009): 1-10. https://doi.org/10.1016/j.amc.2007.05.070

[22] Al-Salti, Nasser, Erkinjon Karimov, and Kishin Sadarangani. "On a differential equation with Caputo-Fabrizio fractional derivative of order $1<\mathrm{b}<2$ and application to mass-spring-damper system." Prog. Fract. Differ. Appl. 2, no. 4 (2016): 257-263. https://doi.org/10.18576/pfda/020403

[23] Sheikh, Nadeem Ahmad, Farhad Ali, Ilyas Khan, and Muhammad Saqib. "A modern approach of Caputo-Fabrizio time-fractional derivative to MHD free convection flow of generalized second-grade fluid in a porous medium." Neural Computing and Applications 30, no. 6 (2018): 1865-1875.

https://doi.org/10.1007/s00521-016-2815-5

[24] Ali, Farhad, Nadeem Ahmad Sheikh, Ilyas Khan, and Muhammad Saqib. "Magnetic field effect on blood flow of Casson fluid in axisymmetric cylindrical tube: A fractional model." Journal of Magnetism and Magnetic Materials 423 (2017): 327-336. https://doi.org/10.1016/i.jmmm.2016.09.125

[25] Abro, Kashif Ali, and J. F. Gomez-Aguilar. "A comparison of heat and mass transfer on a Walter'sB fluid via CaputoFabrizio versus Atangana-Baleanu fractional derivatives using the Fox-H function." The European Physical Journal 
Plus 134, no. 3 (2019): 101.

https://doi.org/10.1140/epjp/i2019-12507-4

[26] Shah, Nehad Ali, and Ilyas Khan. "Heat transfer analysis in a second grade fluid over and oscillating vertical plate using fractional Caputo-Fabrizio derivatives." The European Physical Journal C 76, no. 7 (2016): 362.

https://doi.org/10.1140/epjc/s10052-016-4209-3

[27] Saqib, Muhammad, Ilyas Khan, and Sharidan Shafie. "Application of fractional differential equations to heat transfer in hybrid nanofluid: modeling and solution via integral transforms." Advances in Difference Equations 2019, no. 1 (2019): 1-18.

https://doi.org/10.1186/s13662-019-1988-5

[28] Alkahtani, B. S. T., and A. Atangana. "Controlling the wave movement on the surface of shallow water with the Caputo-Fabrizio derivative with fractional order." Chaos, Solitons \& Fractals 89 (2016): 539-546.

https://doi.org/10.1016/i.chaos.2016.03.012

[29] Ali Shah, Nehad, Najma Ahmed, Thanaa Elnaqeeb, and Mohammad Mehdi Rashidi. "Magnetohydrodynamic free convection flows with thermal memory over a moving vertical plate in porous medium." Journal of Applied and Computational Mechanics 5, no. 1 (2019): 150-161.

[30] Shah, Nehad Ali, Dumitru Vieru, and Constantin Fetecau. "Effects of the fractional order and magnetic field on the blood flow in cylindrical domains." Journal of Magnetism and Magnetic Materials 409 (2016): 10-19.

https://doi.org/10.1016/i.jmmm.2016.02.013

[31] Hatami, M., J. Hatami, and Davood Domiri Ganji. "Computer simulation of MHD blood conveying gold nanoparticles as a third grade non-Newtonian nanofluid in a hollow porous vessel." Computer methods and programs in biomedicine 113, no. 2 (2014): 632-641.

https://doi.org/10.1016/i.cmpb.2013.11.001

[32] Sharma, Mukesh Kumar, Kuldip Singh, and Seema Bansal. "Pulsatile MHD flow in an inclined catheterized stenosed artery with slip on the wall." Journal of Biomedical Science and Engineering 2014 (2014).

https://doi.org/10.4236/ibise.2014.74023

[33] Ali, Farhad, Nadeem Ahmad Sheikh, Ilyas Khan, and Muhammad Saqib. "Magnetic field effect on blood flow of Casson fluid in axisymmetric cylindrical tube: A fractional model." Journal of Magnetism and Magnetic Materials 423 (2017): 327-336.

https://doi.org/10.1016/i.jmmm.2016.09.125 